\title{
Disruption of direct 3D telomere-TRF2 interaction through two molecularly disparate mechanisms is a hallmark of primary Hodgkin and Reed-Sternberg cells
}

\author{
Hans Knecht ${ }^{1}$, Nathalie A Johnson ${ }^{1}$, Tina Haliotis ${ }^{1,2}$, Daniel Lichtensztejn ${ }^{3}$ and Sabine Mai ${ }^{3}$
}

In classical Hodgkin's lymphoma ( $\mathrm{CHL}$ ), specific changes in the 3D telomere organization cause progression from mononuclear Hodgkin cells (H) to multinucleated Reed-Sternberg cells (RS). In a post-germinal center B-cell in vitro model, permanent latent membrane protein 1 (LMP1) expression, as observed in Epstein-Barr virus (EBV)-associated cHL, results in multinuclearity and complex chromosomal aberrations through downregulation of key element of the shelterin complex, the telomere repeat binding factor 2 (TRF2). Thus, we hypothesized that the three-dimensional (3D) telomereTRF2 interaction was progressively disturbed during transition from $\mathrm{H}$ to RS cells. To this end, we developed and applied for the first time a combined quantitative 3D TRF2-telomere immune fluorescent in situ hybridization (3D TRF2/Telo-QFISH) technique to monolayers of primary $\mathrm{H}$ and RS cells, and adjacent benign internal control lymphocytes of lymph node biopsy suspensions from diagnostic lymph node biopsies of 14 patients with $\mathrm{cHL}$. We show that $\mathrm{H}$ and RS cells are characterized by two distinct patterns of disruption of 3D telomere-TRF2 interaction. Disruption pattern A is defined by massive attrition of telomere signals and a considerable increase of TRF2 signals not associated with telomeres. This pattern is restricted to EBV-negative $\mathrm{CHL}$. Disruption pattern $\mathrm{B}$ is defined by telomere de-protection due to an impressive loss of TRF2 signals, physically linked to telomeres. This pattern is typical of, but is not restricted to, LMP1+EBV-associated CHL. In the disruption pattern B group, so-called 'ghost' end-stage RS cells, void of both TRF2 and telomere signals, were identified, whether or not associated with EBV. Our findings demonstrate that two molecularly disparate mechanisms converge on the level of 3D telomere-TRF2 interaction in the formation of RS cells.

Laboratory Investigation (2017) 97, 772-781; doi:10.1038/labinvest.2017.33; published online 24 April 2017

Numerical and complex structural chromosomal aberrations are commonly identified in Hodgkin's lymphoma (HL)derived cell lines ${ }^{1-3}$ and microdissected primary mononuclear cells. The 3D transition from Hodgkin $(\mathrm{H})$ to multinucleated Reed-Sternberg (RS) cells ${ }^{4-6}$ is characterized by dynamic progressive 3D telomere dysfunction, ${ }^{7}$ formation of giant 'zebra' chromosomes, ${ }^{8}$ and remodeling of the nuclear DNA organization. ${ }^{9-11}$ These changes are associated with major changes in the telomere-protecting shelterin complex. ${ }^{12,13}$ Analogous findings are observed in an in vitro model for post-germinal center B-cell, Epstein-Barr virus (EBV)associated classical HL (cHL). ${ }^{14}$ In this experimental BJABtTA-LMP1 tet-Off system, $>90 \%$ of the cells express the EBV-encoded oncogene latent membrane protein 1 (LMP1), in contrast to the KMH2-EBV $\mathrm{H}$ cell subline, where under permanent stimulation with CD40L/IL-4, the LMP1 oncogene is only expressed by $50 \%$ of the cells. ${ }^{15}$ In the BJAB-tTA-LMP1 tet-Off system, LMP1 mediates multinuclearity and complex chromosomal abnormalities primarily through downregulation of telomere repeat binding factor 2 (TRF2). ${ }^{14}$ TRF2 has recently emerged as a key element of the shelterin complex ${ }^{16}$ and also interacts with lamin $\mathrm{A} / \mathrm{C}$ in the maintenance of the $3 \mathrm{D}$ genome organization. ${ }^{17}$ Though TRF2 appears to be the major target of LMP1, other shelterin proteins, such as TRF1 (TRF1) and protection of telomeres 1 (POT1), are also reversibly downregulated through LMP1. ${ }^{14}$ Moreover, EBV-infected normal human B lymphocytes (Ly) show not only partial displacement of TRF2 ${ }^{18}$ but also low levels of TRF2, TRF1, and POT1. ${ }^{19}$

${ }^{1}$ Division of Hematology, Department of Medicine, Jewish General Hospital, McGill University, Montréal, QC, Canada; ${ }^{2}$ Department of Pathology, Jewish General Hospital, McGill University, Montréal, QC, Canada and ${ }^{3} \mathrm{MICB}$, University of Manitoba, Winnipeg, MB, Canada

Correspondence: Professor H Knecht, MD, Division of Hematology, Department of Medicine, Jewish General Hospital, McGill University, 3755, Chemin de la Côte

Ste-Catherine, McGill University, Montréal, QC H3T 1E2, Canada.

E-mail: hans.knecht@mcgill.ca

Received 22 August 2016; revised 22 January 2017; accepted 8 February 2017 
Telomerase activity in cHL was for the first time reported by Brousset et a ${ }^{20}$ in 1997 in only one of eight cases without telomerase inhibition. However, this study was performed on whole- protein extracts, including the bulk of reactive Ly. Two subsequent studies identified human telomerase reverse transcriptase (hTERT) by in situ hybridization (ISH) in $\mathrm{H}$ and RS cells of all HL cases analyzed. ${ }^{21,22}$ However, there was no correlation between the number of hTERT-positive H and RS cells (varying from 30 to $70 \%$ ), the HL subtype, and the LMP1 positivity, ${ }^{22}$ consistent with the possibility that the telomere protecting shelterin complex was as important as the telomerase activity itself.

Thus, we hypothesized that the 3D interaction of telomeres and TRF2 is disrupted in $\mathrm{H}$ cells and directly associated with the formation of $\mathrm{H}$ and RS cells. To this end, we developed a combined quantitative 3D TRF2-telomere immuno Q-FISH (3D TRF2/Telo-Q-FISH) protocol and applied it to monolayers of primary $\mathrm{H}$ and $\mathrm{RS}$ cells including surrounding reactive Ly from diagnostic lymph node biopsy suspensions, allowing 3D analysis of the entire nuclear content (often not achieved using laser microdissection of $\mathrm{H}$ and RS cells, given that their nuclei are generally $>10 \mu \mathrm{m}$ in diameter and that this technique is performed on $5 \mu \mathrm{m}$ sections). Our results show that the 3D steric interaction between telomeres and TRF2 is progressively disrupted from $\mathrm{H}$ to RS cells by one of two different mechanisms. Either, massive attrition of telomeres is associated with an overwhelming increase in TRF2 signals that are no longer associated with telomeres, or marked loss of TRF2 signals physically linked to telomeres result in telomere de-protection. The first pattern of disruption of the 3D telomere-TRF2 interaction is only observed in EBV-negative cases, whereas the second pattern is typical of but not restricted to EBV-positive LMP1-expressing cases. Thus, our in vitro model for post-germinal center B-cell, EBV-associated cHL, where the EBV-encoded oncogene LMP1 mediates multinuclearity through downregulation of TRF2, is confirmed. Most importantly, besides this pathway leading to telomere dysfunction, there is at least one second (opposite) pathway where telomere dysfunction (mainly attrition of telomeres) is associated with TRF2 upregulation. In either pathway, the 3D shelterin complex is qualitatively and quantitatively disrupted.

\section{MATERIALS AND METHODS}

\section{Control Cell Line}

Primary Human BJ-5ta fibroblast cells (ATCC) were grown in bicarbonate-buffered RPMI-1640 medium supplemented with $10 \%$ bovine fetal calf serum, penicillin $(200 \mathrm{U} / \mathrm{ml})$ and streptomycin $(200 \mathrm{mg} / \mathrm{ml})$, and were incubated at $37^{\circ} \mathrm{C}$ in a humidified atmosphere containing $5 \% \mathrm{CO}_{2}$.

Isolation of Primary Hodgkin and Reed-Sternberg Cells Isolation and cryopreservation of primary $\mathrm{H}$ and RS cells was performed according to the Jewish General Hospital SOP \# 8.3.004 version 2 of the BCLQ (Banque de Cellules
Leucémiques du Québec). These guidelines are established respecting the Declaration of Helsinki and approved by the Ethical Committee of the Jewish General Hospital, Montreal, Canada. The detailed protocol is available upon request from the BCLQ. Briefly, fresh tissue from diagnostic lymph nodes was under sterile conditions manually disaggregated in very small 1-2 mm measuring pieces and subsequently further disaggregated using the GentleMACS tissue dissociator. In order to protect the cellular integrity of $\mathrm{H}$ and RS cells, the step for enrichment for B cells using EasySep magnetic columns was omitted, the cellular suspension centrifuged, resuspended at $5 \times 10^{7}$ cells per $\mathrm{ml}$ using freezing medium (10\% DMSO in bovine fetal calf serum), placed in cryovials and snap frozen until used for combined 3D TRF2/Telo-QFISH.

\section{D TRF2/Telo-Q-FISH}

Cryopreserved cellular suspensions of diagnostic lymph nodes were thawed, resuspended in bicarbonate-buffered RPMI-1640 medium supplemented with $10 \%$ bovine fetal calf serum, penicillin $(200 \mathrm{U} / \mathrm{ml})$ and streptomycin $(200 \mathrm{mg} /$ $\mathrm{ml}$ ), and incubated at $37^{\circ} \mathrm{C}$ in a humidified atmosphere containing $5 \% \mathrm{CO}_{2}$ for $12 \mathrm{~h}$ to a maximum of $36 \mathrm{~h}$. Ly, $\mathrm{H}$, and RS cells of $14 \mathrm{cHL}$ were analyzed, 4 of them belonging to EBV-associated HL with LMP1-expressing H and RS cells. Quality of $\mathrm{H}$ and RS cells was assessed by Wright-Giemsa staining on monolayers of cytocentrifuged cells and revealed no cytologic differences between $\mathrm{H}$ and RS cells of EBV-negative and LMP1-expressing cases (Supplementary Figure 1). Occasionally, large reactive macrophages may have the same size as $\mathrm{H}$ cells, but their nuclear structure differs significantly from the nuclear structure of $\mathrm{H}$ cells. On Wright-Giemsa and non-specific esterase-stained (phagocyte-specific cytochemistry) monolayers, these large macrophages have ovoid ore sole-shaped lateralized nuclei with a homogenous chromatin structure and do not contain macronucleoli typical of $\mathrm{H}$ and RS cells (Supplementary Figure 1A). Most importantly, in the combined immuneFISH protocol applied herein, all $\mathrm{H}$ and RS cells were rigorously selected based on their DNA structure (DAPI staining) characterized by DNA-poor and DNA-free regions. The combined quantitative 3D TRF2-telomere immuno Q-FISH protocol, ${ }^{14,23,24}$ further developed for this study is detailed as follows: (A) Immunostaining: rabbit polyclonal TRF2 (1 mg/ml), Novus (NB110 57130) 1:500 dilution. Secondary antibody: goat anti-rabbit Alexa 488, (Molecular Probes), 1:1000 dilution. Staining steps: 1. Fix cells in 3.7\% formaldehyde $/ 1 \times$ PBS, $10 \mathrm{~min}$, room temperature (RT). 2 . Wash in PBS twice, 5 min, RT, shaking. 3. Permeabilize with $0.1 \%$ Triton X-100 (in $\mathrm{ddH}_{2} \mathrm{O}$ ), 12 min, no shaking, RT. 4 . Wash in PBS three times, 5 min, RT, shaking. 5. Block in 4\% $\mathrm{BSA} / 4 \times \mathrm{SSC}$ for $5 \mathrm{~min}$, RT. 6 . Add antibody in $4 \% \mathrm{BSA} /$ $4 \times$ SSC, $45 \mathrm{~min}, 37^{\circ} \mathrm{C}$, humidified atmosphere. 7 . Wash in PBS three times, $5 \mathrm{~min}$, RT, shaking. 8. Add secondary antibody in $4 \% \mathrm{BSA} / 4 \times \mathrm{SSC}, 30 \mathrm{~min}, 37^{\circ} \mathrm{C}$, humidified 
atmosphere. 9. Three washes in PBS, 5 min, RT, shaking. (B) FISH: 1. Apply $8 \mu$ l PNA-telomere probe (DAKO) (in the dark), add $25 \times 25 \mathrm{~mm}$ cover slip, apply rubber cement to seal. 2. Denature $3 \min 80^{\circ} \mathrm{C}$, hybridize $2 \mathrm{~h}$ at $30^{\circ} \mathrm{C}$ (Hybrite, Vysis/Abbott). 3. Remove rubber cement carefully and work in the dark (from now until the end of the protocol). 4. Place slides including cover slips in $70 \%$ formamide $/ 10 \mathrm{mM}$ Tris $(\mathrm{pH}=7.4)$ shaking until cover slip floats off; wash twice $15 \mathrm{~min}$ in this solution after cover slip is removed, RT, shaking. 5. Wash in $1 \times \mathrm{PBS}$, RT, $1 \mathrm{~min}$, shaking. 6 . Wash $5 \mathrm{~min}$ at $55^{\circ} \mathrm{C}$ in $0.1 \times \mathrm{SSC}$, shaking. 7 . Wash $2 \times 5 \mathrm{~min}$ in $2 \times \mathrm{SSC} / 0.05 \%$ Tween 20 , RT, shaking. 8 . Re-apply primary and secondary antibodies as described above. 9. Wash three times in $1 \times$ PBS. 10 . Stain with DAPI $(0.1 \mathrm{mg} / \mathrm{ml}$ stock $)$, apply $50 \mu \mathrm{l}$, cover with cover slip, incubate in the dark for 3 min. 11. Mount in Vectashield. 12. Image. Imaging of interphases after combined quantitative 3D TRF2-telomere immuno Q-FISH was performed by using Zeiss Axiomanager Z2 with a cooled AxioCam HR B\&W, DAPI, Cy3 (telomere) and FITC (TRF2) filters in combination with a Planapo $\times 63 / 1.4$ oil objective lens. Images were acquired by using AXIOVISION 4.8 (Zeiss) in multichannel mode followed by constraint iterative deconvolution as specified below.

\section{D Image Acquisition}

Interphase nuclei of BJ-5ta control fibroblasts, internal control Ly, mononucleated $\mathrm{H}$ cells, or bi/multinucleated RS cells were analyzed. AXIOvisIon 4.6 with deconvolution module and rendering module were used. For every fluorochrome, the 3D image consists of a stack of 80 images with a sampling distance of $200 \mathrm{~nm}$ along the $z$ and $102 \mathrm{~nm}$ in the $x, y$ direction. The constrained iterative algorithm option was used for deconvolution. ${ }^{25}$ For statistical analysis the number of telomeres was plotted against the intensity of telomere signals (telomere length) and the number of TRF2 signals against the intensity of TRF2 signals. A minimum of 30 interphase nuclei was analyzed.

\section{D Image Analysis for Telomeres}

Telomere measurements were done with TeloView. ${ }^{26}$ By choosing a simple threshold for the telomeres, a binary image is found. On the basis of that, the center of gravity of intensities is calculated for every object resulting in a set of coordinates $(x, y, z)$ denoted by crosses on the screen. The integrated intensity of each telomere is calculated because it is proportional to the telomere length. ${ }^{26}$

\section{Telomere Aggregates}

Telomere aggregates are defined as clusters of telomeres that are found in close association and cannot be further resolved as separate entities at an optical resolution limit of $200 \mathrm{~nm} .{ }^{27}$

\section{Telomere Length}

Telomeres with a relative fluorescent intensity ( $x$ axis) ranging from 0 to 5000 units are classified as very short, with an intensity ranging from 5000 to 15000 units defined as short, with an intensity ranging from 15000 to 30000 units defined as mid-sized, and with an intensity $>30000$ units defined as large. ${ }^{28}$

\section{Telomere Volume}

Total telomere volume is the sum of all very short, short, mid-sized, and large telomeres and aggregates within one mononuclear or multinucleated cell.

\section{Nuclear Volume}

The nuclear volume is calculated according to the $3 \mathrm{D}$ nuclear 4',6-diamidino-2-phenylindoline staining as described earlier. ${ }^{29}$

\section{D Image Analysis of TRF2 Spots}

The measurement parameters applied for the TRF2 length and TRF2 volume were identical to those established for telomeres.

\section{Statistical Analysis}

For each situation, where 30 cells (Ly, $\mathrm{H}$ cells, and RS cells) were analyzed, normally distributed parameters are compared between the two types of cells using nested ANOVA or twoway ANOVA. Multiple comparisons using the least square means tests followed, in which interaction effects between two factors were found to be significant. Other parameters that were not normally distributed were compared using a nonparametric Wilcoxon rank sum test. Significance level was set at $P=0.05$. Analyses were carried out using SAS v9.1 programs.

\section{RESULTS}

\section{Clinical Data}

Table 1 summarizes the clinical data of the 14 patients, where monolayers of primary entire $\mathrm{H}$ and $\mathrm{RS}$ as well as internal control Ly from diagnostic lymph node biopsy suspensions were available for the 3D combined quantitative 3D TRF2telomere immuno Q-FISH (3D TRF2/Telo-Q-FISH) protocol. This approach allowed for the first time 3D analysis of the entire nuclear content of primary $\mathrm{H}$ and RS. Of note, this entire nuclear analysis is not achieved when using laser microdissection of $\mathrm{H}$ and RS, given that their nuclei are generally $>10 \mu \mathrm{m}$ in diameter and that this technique is performed on $5 \mu \mathrm{m}$ sections.

\section{Control Cell Line and Internal Controls}

Most of the BJ-5ta normal human diploid control fibroblasts and most internal control Ly of both LMP1-positive and LMP1-negative cases showed a quantitatively and qualitatively intact 1:1, tight direct 3D association of telomeres with TRF2 (Figure 1) composed mainly of short and intermediate length telomeres, whereas the number of very-short-telomeres ( $\leq 5000$ units) was low: BJ-5ta 16.7\%; case 2 Ly 19.8\%; case 4 Ly $20.8 \%$; case 7 Ly $8.7 \%$ (Figure 1). Corresponding 
Table 1 Clinical data of patients with 3D analysis of primary $H$ and RS cells

\begin{tabular}{|c|c|c|c|c|c|}
\hline Case/Sex & Age & cHL-type & Stage & First-line treatment & Outcome \\
\hline $1 \mathrm{~F}$ & 23 & NS LMP1+ & IB & $3 \times A B V D+I F R T$ & $C R$ \\
\hline $2 F$ & 55 & NS & IVA & $6 \times A B V D$ & Progression \\
\hline $3 M$ & 38 & NS & IVB & $4 \times A B V D+4 \times A V D$ & $C R$ \\
\hline $4 \mathrm{M}$ & 85 & NS & 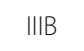 & $6 \times$ ChIVPP & Progression \\
\hline $5 \mathrm{M}$ & 21 & NS & $\| \mathrm{B}$ & $6 \times A B V D$ & $C R$ \\
\hline $6 \mathrm{M}$ & 37 & MC LMP1+ & IA & $3 \times A B V D$ & $C R$ \\
\hline $7 \mathrm{M}$ & 29 & MC LMP1+ & $\| \mathrm{A}$ & $4 \times A B V D$ & $C R$ \\
\hline $8 M$ & 50 & $M C$ & $\| I I A$ & $6 \times A B V D$ & $C R$ \\
\hline $9 \mathrm{~F}^{\mathrm{a}}$ & 58 & $M C$ & IVB & $6 \times A B V D$ & Relapse \\
\hline $10 \mathrm{M}$ & 47 & NS LMP1+ & IA & $2 \times A B V D$ & $C R$ \\
\hline $11 \mathrm{M}$ & 19 & $M C$ & $\| \mathrm{A}$ & $4 \times A B V D$ & $C R$ \\
\hline $12 \mathrm{M}$ & 31 & NS & IVA & $6 \times A B V D$ & $C R$ \\
\hline $13 \mathrm{M}$ & 39 & NS & $\| \mathrm{A}$ & $4 \times A B V D$ & Progression \\
\hline $14 \mathrm{~F}$ & 75 & NS & IVA & $6 \times A B V D+I F R T$ & Progression \\
\hline
\end{tabular}

Abbreviations: ABVD, doxorubicin, bleomycin, vinblastine, dacarbazine; AVD, doxorubicin, vinblastine, dacarbazine; ChIVPP, chlorambucil, vinblastine, procarbazine, prednisone; IFRT, involved field radiation therapy; LMP1+, latent membrane protein 1 positive; MC, mixed cellularity classical Hodgkin lymphoma; NS, nodular sclerosis classical Hodgkin lymphoma.

aiopsy at relapse.

findings were identified for the very-short-TRF2 spots $(\leq 5000$ units), namely BJ-5ta 23.6\%; case 2 Ly $10.5 \%$; case 4 Ly 31.7\%; case 7 Ly $11.0 \%$ (Figure 1).

\section{$H$ and RS Cells}

The $\mathrm{H}$ and RS cells of all 14 cases diagnosed with cHL (Table 1) consistently displayed quantitative and qualitative 3D telomere-TRF2 dissociation, with a significant increase $(P<0.0001)$ of very-short-telomeres (case $8 \mathrm{H}$ cells $46.6 \%$; case $10 \mathrm{H}$ cells $74.3 \%$; case $11 \mathrm{H}$ cells $47.6 \%$; case 2 RS cells $58.9 \%$; case 11 RS cells $94.4 \%$ ) compared to the internal control Ly and the BJ-5ta control cell line. Interestingly, two opposite disruption patterns of the 3D telomere-TRF2 interaction were identified.

\section{Disruption Pattern A}

Massive attrition of telomere signals with a considerable increase of TRF2 signals not associated with telomeres was restricted to six EBV-negative HL (Figure 2) including three clinically aggressive cases (cases 2, 13, and 14). When compared to $\mathrm{H}$ cells (Figure 2a) increasing numbers of free TRF2 signals and further attrition of telomere signals with a relative increase of very-short-telomeres were found in RS cells (Figure 2b). A TeloView analysis of TRF2 and telomere dynamics characteristic for the disruption pattern A are shown in Figure 3. DNA bridges between nuclei of RS cells were identified in two of these cases (Supplementary Figure 2).

\section{Disruption Pattern B}

Telomere de-protection due to a loss of TRF2 signals physically linked to telomeres was identified in eight cases including all four cases of EBV-associated cHL. In particular, two of the four LMP1-expressing cases were characterized by numerous telomeres without TRF2 interaction (de-protected) and this attrition of TRF2 signals was progressive from $\mathrm{H}$ to RS cells (Figures $4 \mathrm{a}$ and $\mathrm{b}$ ). These findings in primary $\mathrm{H}$ and RS cells are analogous to the results of our in vitro model for the transition from $\mathrm{H}$ to RS cells confirming our hypothesis of TRF2 downregulation as a major element for this transition in EBV-associated CHL. However, though this disruption pattern is characteristic for $\mathrm{EBV}$-associated $\mathrm{cHL}$, it is not restricted to it and presents as the opposite of disruption pattern $\mathrm{A}$ (Figure 5). In this group (disruption pattern B) so-called 'ghost' end-stage RS cells, void of both, TRF2 and telomere signals, were identified, whether or not associated with EBV (Figure 6).

In summary, our results show that primary $\mathrm{H}$ and $\mathrm{RS}$ of all $14 \mathrm{cHL}$ cases are characterized by a disruption of the quantitative and qualitative 3D telomere/TRF2 interaction (Table 2). This disruption results in double-stranded DNA breaks, which increase from $\mathrm{H}$ to RS cells as evidenced by $\gamma$-H2AX-positive staining independently of LMP1 expression (Supplementary Figure 3). Two different scenarios appear to be involved. In one scenario (Disruption pattern B), present in all four cases of EBV-associated, LMP1-expressing cHL, a significant downregulation of TRF2 occurs in accordance with our in vitro, LMP1 driven, model of EBV-associated cHL. This scenario is also identified in some EBV-negative cases of cHL. In the other scenario (Disruption pattern A), massive attrition of telomeres is associated with TRF2 upregulation. This pattern was identified in EBV-negative cases of cHL and may be related to clinically aggressive disease.

\section{DISCUSSION}

\section{Recapitulation of the 3D Main Findings}

Our combined qualitative and quantitative 3D telomere-TRF2 analysis performed on complete nuclei of primary $\mathrm{H}$ and RS cells from diagnostic pre-treatment lymph node biopsies reveals a hitherto unknown disruption of the physiological 3D telomere-TRF2 interaction. Surprisingly, two opposite types of this disruption pattern were identified. The first one (Disruption pattern A) characterized by a significant TRF2 upregulation and attrition of telomere signals, the second one (Disruption pattern B) by a massive reduction of TRF2 signals compared to the telomere signals. In both types TRF2 is not bound to all telomeres but the internal control Ly mostly show a preserved quantitative and qualitative 3D telomereTRF2 interaction, thereby excluding technical artifacts as the basis of the observed highly significant differences. 


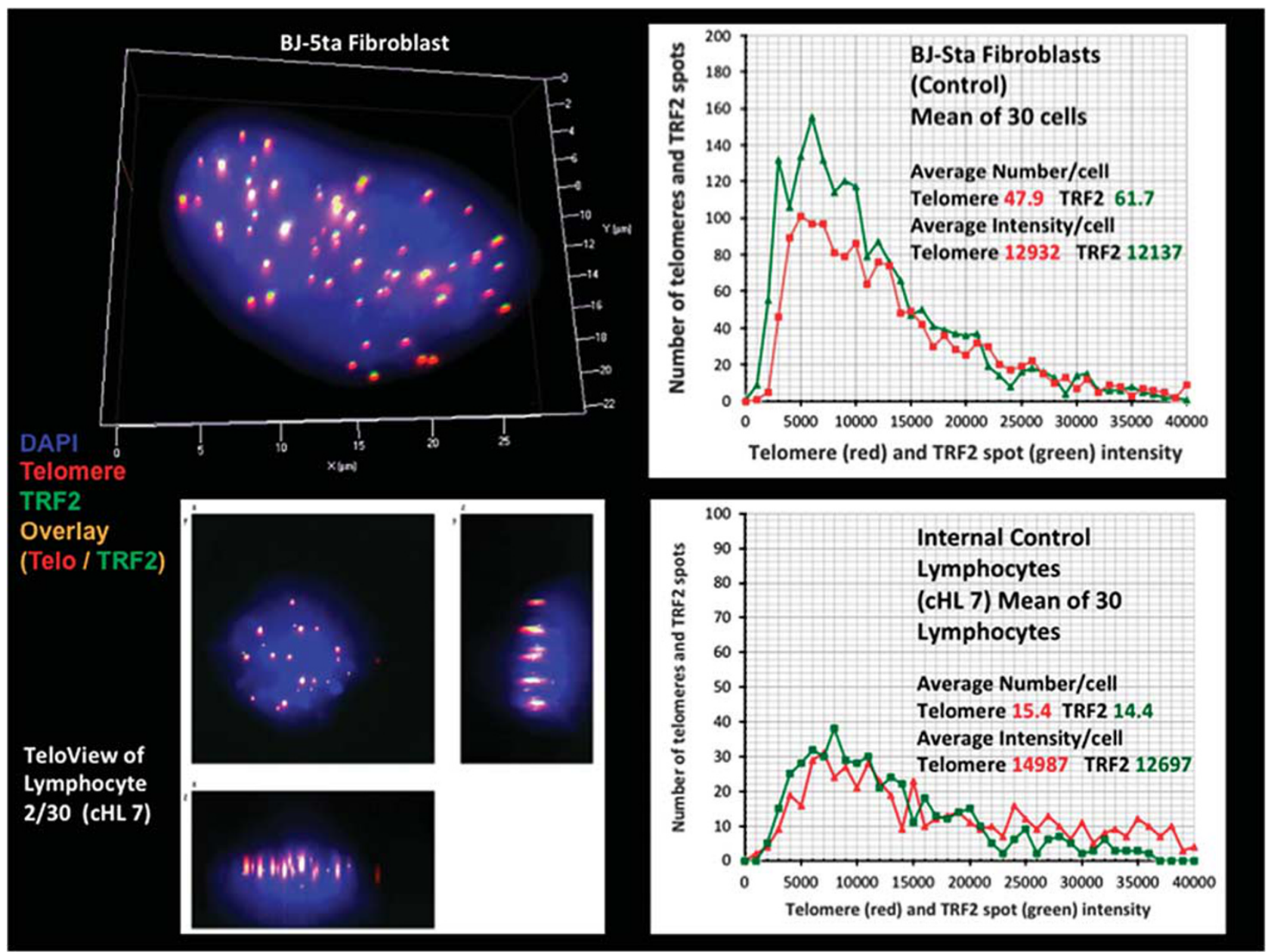

Figure 1 Non-malignant control cells for 3D TRF2/Telo-Q-FISH: the BJ-5ta human fibroblast cell line served as non lymphoid controls and randomly chosen lymphocytes of monolayers of every case served as internal controls. 3D nuclear organization of total nuclear DNA (blue), telomeres (red), TRF2 (green), and telomere-TRF2 overlay (orange). Upper panel: complete 3D reconstitution of a B-5ta human fibroblast with preserved 1:1 direct interaction of telomere and TRF2 signals with equal intensity (left). TeloView analysis of $30 \mathrm{BJ}$-5ta fibroblasts (right). Frequency ( $x$ axis) is plotted against relative fluorescent intensity, ie, size of telomeres/TRF2 spots ( $y$ axis). Lower panel: Teloview analysis of lymphocytes. The telomere signals (white crosses) are marked in three axis $(x, y, z)$ and overlay with the TRF2 spots at a 1:1 ratio (left). TeloView assessment of telomere/TRF2 profiles based on 3D analysis of 30 entire lymphocyte nuclei is shown (right). TRF2, telomere repeat binding factor 2 .

\section{Multiple Facets of TRF2 Function Result in Genome Instability}

TRF2 has recently emerged as a key element of the shelterin complex (reviewed in Feuerhahn et $a^{16}$ ). TRF2 topologically stabilizes the 3 ' single-stranded DNA overhang through DNA wrapping at t-loops, ${ }^{30}$ and is also involved in the formation of t-loops at interstitial telomere repeat sequences that associate with lamin $\mathrm{A} / \mathrm{C},{ }^{17}$ primordial in the maintenance of $3 \mathrm{D}$ genome organization. ${ }^{31}$ Moreover, elevated levels of TRF2 induce telomeric anaphase bridges and rapid telomere deletions emphasizing the importance of TRF2 for the interaction of 3D nuclear structure and function. ${ }^{32}$ Increased levels of TRF2 are found in colorectal carcinoma tissue, ${ }^{33}$ breast cancer cells including their derived cell lines ${ }^{34}$ and adult T-cell leukemia cells. ${ }^{35}$ It has also been shown that TRF2-mediated upregulation of the heparan sulfate (glucosamine) 3-O-sulphotransferase 4 gene in tumor cells decreased their ability to recruit and activate natural killer cells. ${ }^{36}$ On the other hand it is well-known that conditional TRF2 deletion elicits an ATM-mediated telomere damage response with gamma-H2AX upregulation resulting in telomere fusions and consequently giant chromosomes in mouse fibroblast ${ }^{37}$ as well as in endoreplication and giant hepatocytes. ${ }^{38,39}$ Thus, TRF2 expression is essential to avoid nonhomologous end-joining recombination leading to giant chromosomes, hyperploidy, and endomitosis. ${ }^{37,40}$ In mice, targeted deletion of TRF2 in type 2 alveolar epithelial cells results in a severe localized macrophage and T lymphocytedominated inflammatory response triggered by IL-15 and death receptor signaling, ${ }^{41}$ and TRF2 downregulation at the transcriptional and translational level leading to telomere deprotection is also induced by miR-23a overexpression. ${ }^{42}$ 

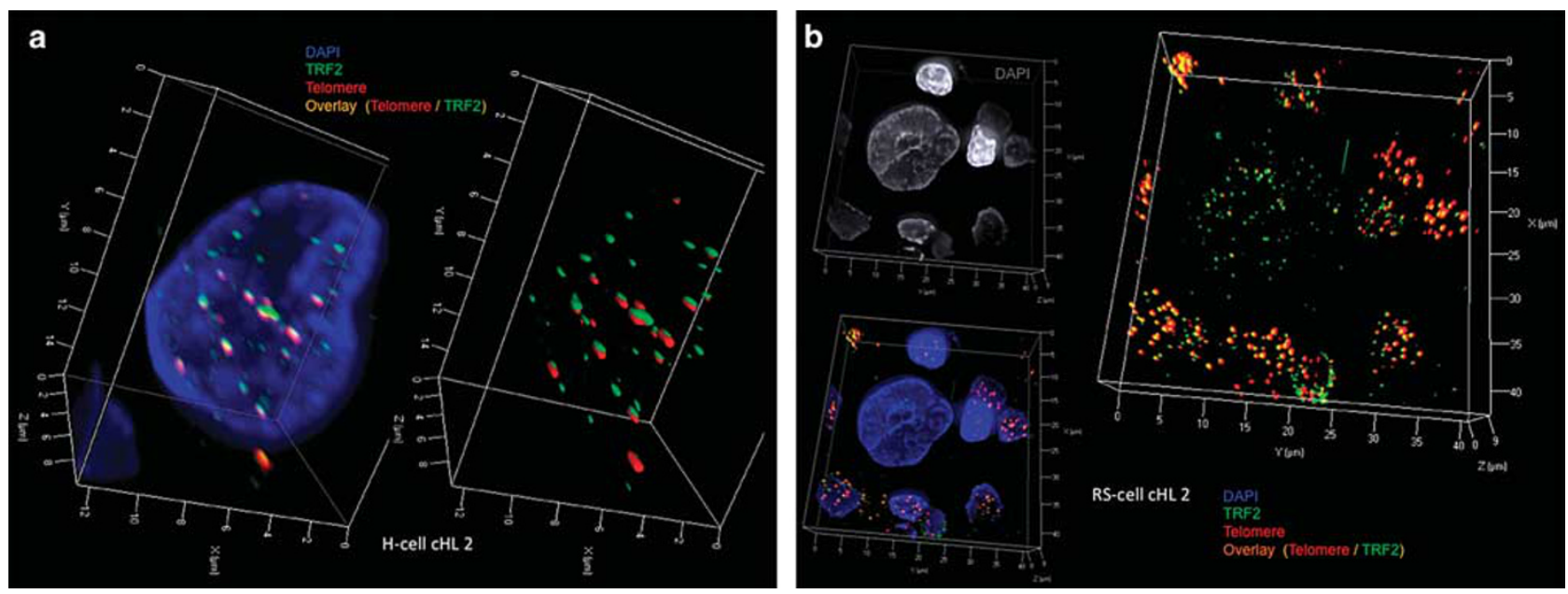

Figure 2 3D telomere/TRF2 disruption pattern A: progressive shortening and loss of telomeres but increase of unbound (free) TRF2 spots occurs during the transition from $\mathrm{H}$ to RS cells in aggressive $\mathrm{CHL}$ (patient 2) (a). Complete 3D reconstitution of mononuclear $\mathrm{H}$ cell (left) with nuclear DNA (blue), telomere (red), TRF2 (green), and telomere-TRF2 overlay (orange) signals is shown in transparency mode. Unbound TRF2 signals and variable telomere/ TRF2 complexes are identified. 3D telomere-TRF2 reconstitution in surface mode of same nucleus (right panel) confirms numerous unbound (free) TRF2 signals and variation of the remaining 3D telomere/TRF2 complexes, ie, large TRF2 spots associate with small telomeres and vice versa. (b) Large binuclear RS cell (same patient as in a) shows progressive attrition of telomeres, increase of free TRF2 signals, and unbalanced signal distribution between the two adjacent nuclei. Upper left shows DNA structure of the RS cell and surrounding lymphocytes. Lower left shows 3D nuclear organization of total nuclear DNA (blue), telomeres (red), TRF2 (green), and telomere-TRF2 overlay (orange) in transparency mode. Right panel shows 3D telomere-TRF2 reconstitution in surface mode and confirms telomere attrition, few remaining small telomere signals mainly in the upper nucleus and a lower nucleus nearly void of telomeres. To note that the lymphocyte adjacent to the right side of the RS cell contains also several free TRF2 signals (lymphocyte precursor of $\mathrm{H}$ cell?), several lymphocytes of this type have been identified in this patient. CHL, classical Hodgkin's lymphoma; $\mathrm{H}$, Hodgkin cells; RS, Reed-Sternberg cells; TRF2, telomere repeat binding factor 2.

3D Telomere / TRF2 Kinetics from $\mathrm{H} \rightarrow \mathrm{RS}$ cells in EBV negative $\mathrm{CHL}$

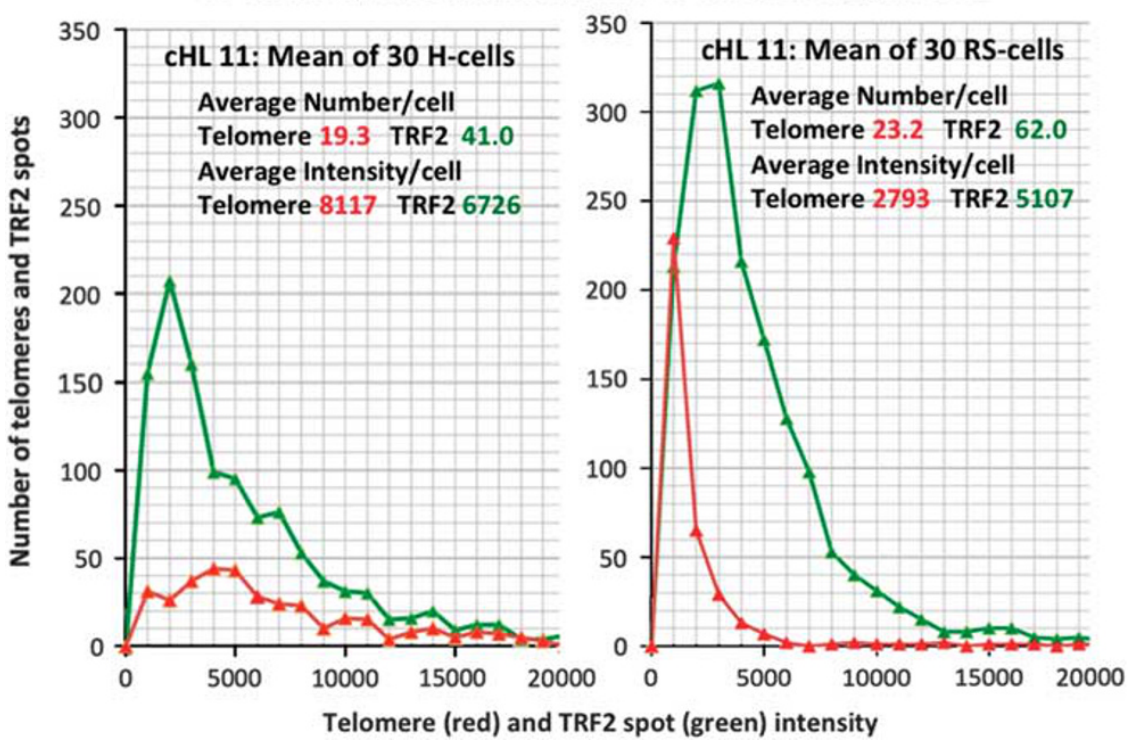

Figure 3 Increased free TRF2 signals in 3D telomere/TRF2 disruption pattern A: telomere (red) and TRF2 spot (green) distribution according to size in mononuclear Hodgkin (left) and at least binuclear Reed-Sternberg cells (right) in the EBV-negative case 11. TeloView results are based on 3D analysis of 30 Hodgkin and 30 Reed-Sternberg cells. Frequency ( $x$ axis) is plotted against relative fluorescent intensity, ie, size of telomeres and TRF2 spots ( $y$ axis). There is a significant shift $(P<0.0001)$ of short telomeres to very-short-telomeres of $\leq 5.000$ relative fluorescence units $(47.6 \%$ in $\mathrm{H}$ cells versus $94.4 \%$ in RS cells) with the total number of telomeres remaining nearly the same (19.3 telomere per $\mathrm{H}$ cell versus 23.2 telomere per RS cell). There is also a significant shift $(P<0.0001)$ to very-short-TRF2 spots $(63.3 \%$ in $\mathrm{H}$ cells versus 73.2 in RS cells) but furthermore paralleled by an increasing number of TRF2 spots (41.0 TRF2 spots per H cell versus 62.0 TRF2 spots per RS cell) documenting the substantial increase of unbound TRF2 signals. EBV, Epstein-Barr virus; H, Hodgkin cells; RS, Reed-Sternberg cells; TRF2, telomere repeat binding factor 2. 

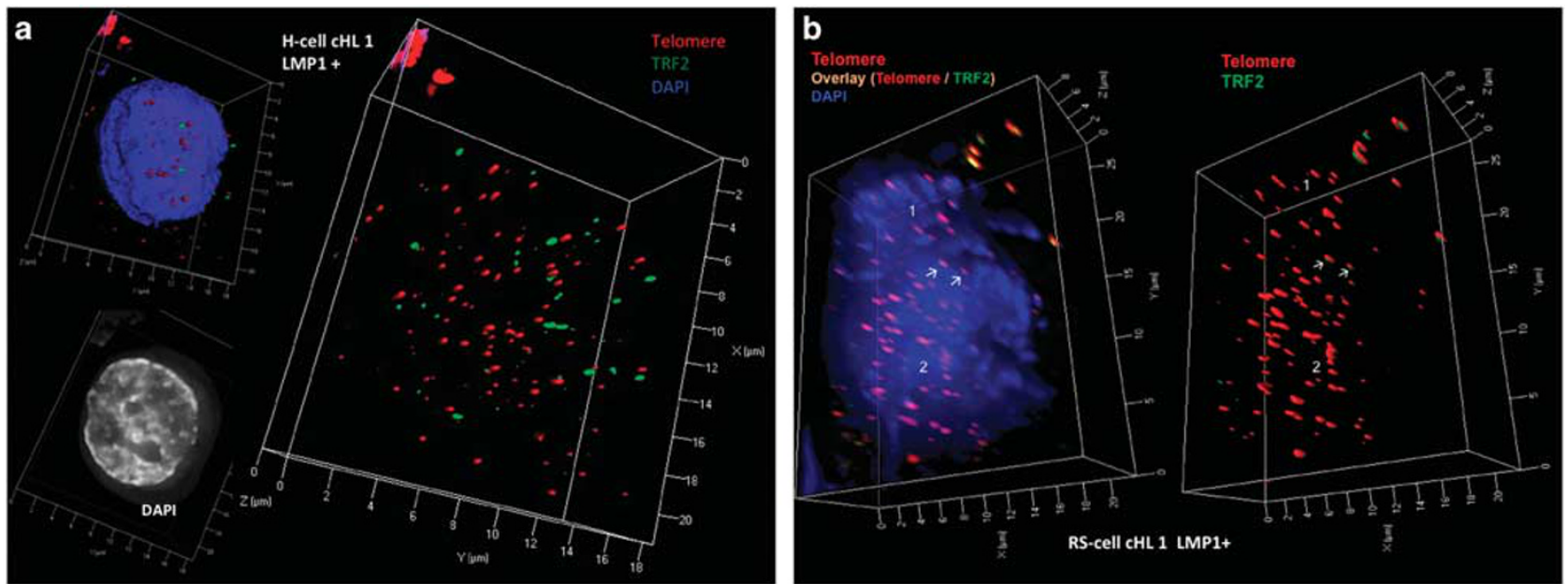

Figure 4 3D telomere de-protection in EBV-associated LMP1-expressing CHL (disruption pattern B): progressive attrition of TRF2 spots occurs in LMP1-positive $\mathrm{CHL}$ during the transition from $\mathrm{H}$ to RS cells. (a). Large mononuclear Hodgkin cell (upper left) shown in 3D surface mode with telomere (red) and few TRF2 spots (green) visible at the surface of the nucleus (blue). Same cell (lower left) in transparency mode for DAPI only to demonstrate typical DNA pattern of a Hodgkin cell. Same $\mathrm{H}$ cell (right panel) in surface mode for telomere (red) and TRF2 spots (green) reveals telomere deprotection due to attrition of TRF2 signals. (b). Left panel showing binuclear $(1,2)$ RS cell (DAPI in blue) in transparency mode with numerous telomere (red) and nearly absent telomere-bound TRF2 (orange) indicated by arrows. Surface mode for telomere (red) and TRF2 (green) of the same cell without DNA staining confirms very few minimal TRF2 signals (green) bound to telomere (arrows). CHL, classical Hodgkin's lymphoma; EBV, Epstein-Barr virus; $\mathrm{H}$, Hodgkin cells; LMP1, latent membrane protein 1; RS, Reed-Sternberg cells; TRF2, telomere repeat binding factor 2.

Two (opposite) Types of 3D Telomere / TRF2 Disruption occur in CHL

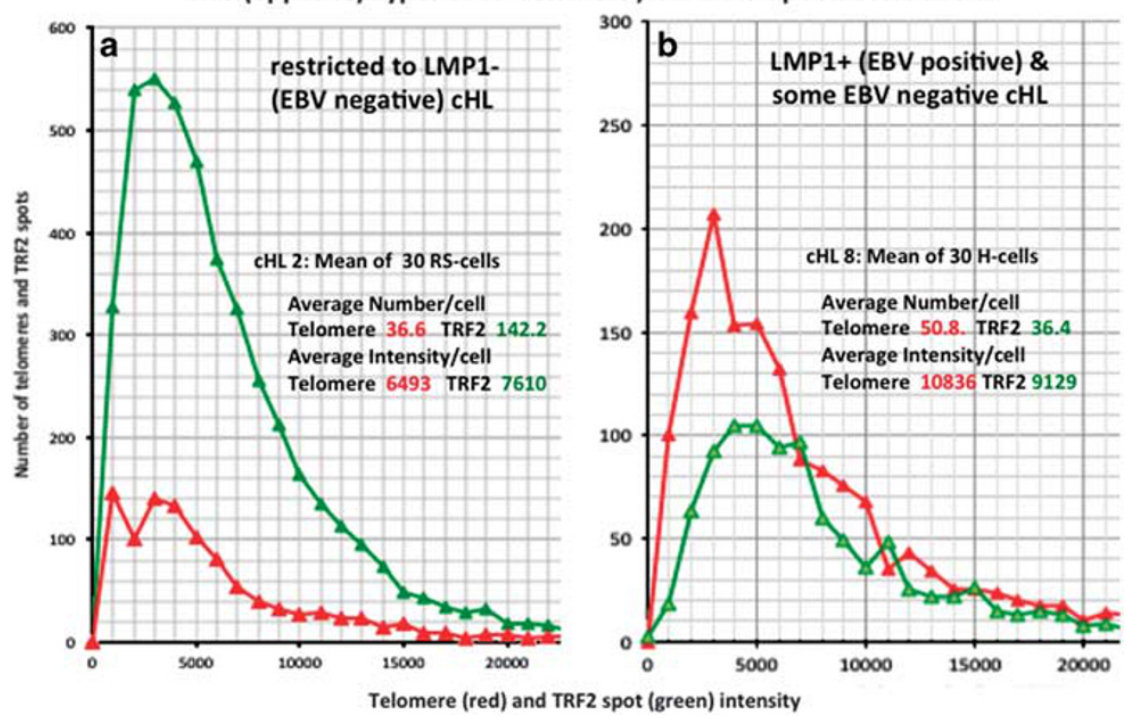

Figure 5 Two opposite ways of 3D telomere/TRF2 disruption. TeloView performed on groups of $30 \mathrm{H}$ and 30 RS cells confirms the findings obtained through visual analysis of cases with $<30 \mathrm{H}$ and/or $30 \mathrm{RS}$ cells. On the left the disruption pattern A with significant increase of TRF2 signals and attrition of telomere signals (either free or still bound to TRF2; see also Figure 2, same case) observed in case 2 is shown. On the right disruption pattern B with TRF2 attrition but still significantly more telomere is shown. Note that this case (case 8 ) is not EBV-associated. In two EBV-associated, LMP1-positive cases ( $\mathrm{CHL}$ 1, Figures $3 a$ and b) and CHL 10 (Table 2), the TRF2 attrition is even much more pronounced. The total number of very-shorttelomere signals is significantly elevated in disruption pattern $B$ compared to $A(P \leq 0.0001)$, whereas in disruption pattern $A$ the total number of very-short-TRF2 spots is significantly increased compared to disruption pattern $B(P \leq 0.0001)$. CHL, classical Hodgkin's lymphoma; EBV, Epstein-Barr virus; $\mathrm{H}$, Hodgkin cells; LMP1, latent membrane protein 1; RS, Reed-Sternberg cells; TRF2, telomere repeat binding factor 2.

Considering these data it is not astonishing that either TRF2 upregulation or TRF2 depletion, both, appear as candidate drivers in the formation of $\mathrm{H}$ and $\mathrm{RS}$ cells, since both scenarios result in telomere de-protection, deregulated immune responses, and genome instability as identified in $\mathrm{cHL}^{43,44}$ even though through different mechanisms. 


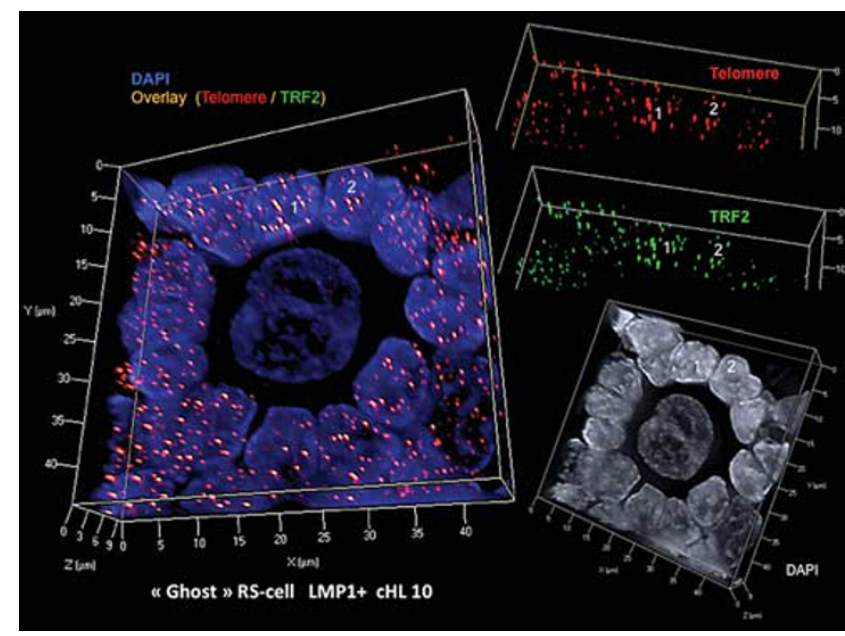

Figure 6 Ghost cells observed associated with disruption pattern B. There is a complete loss of telomere and TRF2 spots in a binucleated, LMP1expressing RS cell, whereas the surrounding lymphocytes show a normal 3D telomere/TRF2 interaction. Lower right is a DAPI staining in transparency mode to optimize morphological aspects. Two lymphocytes $(1,2)$ are identified. Upper right (telomere) and middle right (TRF2) demonstrate that the surrounding lymphocytes $(1,2)$ contain mainly small to mid-sized telomere with a preserved 1:1 3D interaction with TRF2. The left panel (transparency mode) reveals a true ghost RS cell without any telomere and without any TRF2 signals. The lymphocyte corona serves as an internal control. Ghost RS cells (no telomere/no TRF2) were also identified in case 8 . In case 2 (disruption pattern A) RS cells with very few telomere signals but still numerous TRF2 spots were identified. LMP1, latent membrane protein 1; RS, Reed-Sternberg cells; TRF2, telomere repeat binding factor 2 .

\section{Disruption Pattern A}

Very-short-telomeres, so-called 't-stumps,' a hallmark of tumor cells, ${ }^{45}$ were most prominent in the RS cells of this group. Telomeres were either largely reduced or no longer detected by Q-FISH in this category (Figure 2b). A total of six cases showed this pattern, and in three cases with high TRF2 levels (cases 2, 5, and 14) DNA bridges between the nuclei of RS cells were identifiable. This is of particular interest since overexpression of TRF2 in HT1080 human fibrosarcoma cells rapidly leads to telomeric replication stalling, chromosome end-to-end fusions, and loss of telomeric sequences. ${ }^{32}$ In this study, already after the first cell division after TRF2 overexpression ultrafine telomeric anaphase bridges were observed, followed by significant telomere shortening after three to four cell divisions. ${ }^{32}$ This telomere shortening appears to result from a stochastic loss of large telomeric sequences. This experimental model of TRF2 overexpressioninduced telomere deletions and chromosome fusions may explain, our, at a first glance paradoxical-appearing observation of TRF2 overexpressing, telomere poor, DNA bridges containing RS cells, which are characteristic of disruption pattern A.

\section{Disruption Pattern B}

We recently described an experimental in vitro system, which may help to understand the pathogenesis of EBV-associated,
Table 2 Quantitative 3D telomere/TRF2 interaction data

\begin{tabular}{|c|c|c|c|c|c|c|c|c|}
\hline \multirow[t]{2}{*}{ Case } & \multirow[t]{2}{*}{ LMP1 +/- } & \multicolumn{3}{|c|}{$\begin{array}{l}\text { 3D cell analysis } \\
(n)\end{array}$} & \multicolumn{3}{|c|}{$\begin{array}{l}\text { Signal-ratio } \\
\text { telomere/TRF2 }\end{array}$} & \multirow{2}{*}{$\begin{array}{l}\text { Disruption } \\
\text { Pattern A/B }\end{array}$} \\
\hline & & $\mathrm{H}$ & $\mathrm{RS}$ & Ly & $\mathrm{H}$ & RS & Ly & \\
\hline 1 & + & 7 & 5 & 5 & $2.5^{\mathrm{a}}$ & $3.8^{\mathrm{a}}$ & $0.8^{a}$ & B \\
\hline 2 & - & 8 & $30^{\mathrm{b}}$ & $30^{\mathrm{b}}$ & $0.4^{a}$ & $0.26^{\mathrm{b}}$ & $0.64^{\mathrm{b}}$ & A \\
\hline 3 & - & 16 & 4 & 5 & $1.1^{\mathrm{a}}$ & $1.6^{\mathrm{a}}$ & $1.2^{\mathrm{a}}$ & B \\
\hline 4 & - & 9 & 3 & $30^{b}$ & $1.5^{\mathrm{a}}$ & $1.5^{\mathrm{a}}$ & $1.22^{b}$ & B \\
\hline 5 & - & 24 & 21 & 5 & $0.3^{\mathrm{a}}$ & $0.2^{\mathrm{a}}$ & $0.9^{\mathrm{a}}$ & A \\
\hline 6 & + & 6 & 2 & 0 & $1.0^{\mathrm{a}}$ & $1.2^{\mathrm{a}}$ & & B \\
\hline 7 & + & 8 & 2 & $30^{b}$ & $1.4^{\mathrm{a}}$ & $1.5^{\mathrm{a}}$ & $1.07^{\mathrm{b}}$ & B \\
\hline 8 & - & $30^{\mathrm{b}}$ & 3 & 5 & $1.40^{\mathrm{b}}$ & $1.8^{\mathrm{a}}$ & $1.0^{\mathrm{a}}$ & B \\
\hline 9 & - & 5 & 5 & 5 & $2.0^{a}$ & $3.0^{\mathrm{a}}$ & $1.2^{\mathrm{a}}$ & B \\
\hline 10 & + & $30^{b}$ & 8 & 15 & $5.80^{\mathrm{b}}$ & $6.0^{\mathrm{a}}$ & $1.1^{\mathrm{a}}$ & B \\
\hline 11 & - & $30^{\mathrm{b}}$ & $30^{\mathrm{b}}$ & 5 & $0.47^{\mathrm{b}}$ & $0.37^{b}$ & $1.0^{\mathrm{a}}$ & A \\
\hline 12 & - & 6 & 3 & 5 & $0.6^{a}$ & $0.4^{\mathrm{a}}$ & $1.1^{\mathrm{a}}$ & A \\
\hline 13 & - & 6 & 4 & 0 & $0.5^{\mathrm{a}}$ & $0.2^{\mathrm{a}}$ & & A \\
\hline 14 & - & 8 & 2 & 2 & $0.7^{\mathrm{a}}$ & $0.3^{\mathrm{a}}$ & $0.9^{a}$ & A \\
\hline
\end{tabular}

Abbreviations: H, Hodgkin cell; Ly, Lymphocyte; $n$, number of cells analyzed; RS, Reed-Sternberg cell.

${ }^{a}$ Analysis done by visual counting of $3 \mathrm{D}$ pictures (results analogous to results obtained by TeloView).

${ }^{\mathrm{b}}$ Analysis by TeloView (30 cells).

LMP1-expressing HL. ${ }^{14}$ In this model LMP1 induced downregulation of shelterin proteins, especially TRF2, induces TRF2-poor multinucleated cells. Progressive loss of TRF2 signals was observed during the transition of $\mathrm{H}$ to RS cells in all four LMP1-expressing cHL cases of the current study. Moreover, there was a significant shift from short to veryshort-telomeres, so-called 't-stumps.' 45 'Ghost' RS cells, as earlier described ${ }^{7,28}$ were confirmed and these findings were extended to absence of TRF2. Thus, the results presented here appear to be a proof-of-principle that LMP1 expression is a driving element in the formation of $\mathrm{H}$ and RS cells in EBVassociated $\mathrm{CHL}^{14}$ This appears to be a multistep process initiating early after EBV infection of B Ly by EBV through LMP1 targeting the shelterin telomere interaction through both, displacement of TRF2 from the telomeres ${ }^{18}$ and profound TRF2 downregulation. ${ }^{19}$ LMP1 expression itself is sustained by accumulation of reactive oxygen species, which selectively inhibit mRNAs targeting LMP1 transcripts. ${ }^{46}$ However, TRF2 downregulation at the transcriptional and translational level leading to telomere de-protection is also directly induced by miR-23a overexpression. ${ }^{42}$ Thus, miR-23 or further, still unknown downstream effectors, could further account for the telomere de-protection of the four EBVnegative cHL cases presenting the disruption pattern B. Both, the 'ghost' RS cells of LMP1-expressing and LMP1-negative 
cHL appear to represent end stages of a spectrum of changes of nuclear DNA organization revealed by 3D super resolution microscopy (3D SIM) progressing from Ly over mononuclear $\mathrm{H}$ cells to binucleated or finally multinucleated RS cells with DNA anaphase bridges. ${ }^{10}$ These 'ghost' cells ${ }^{28}$ are probably important, since their proteomics machinery is still actively working and thus contributing to the inflammatory (B symptoms) and immunological components of cHL. ${ }^{47-50}$

In summary, the findings presented here appear to identify two different mechanisms leading to the formation of $\mathrm{H}$ and RS cells. The shelterin protein TRF2 may act as a key element in the formation of $\mathrm{H}$ and RS cells. Since TRF2 also interacts with lamin $\mathrm{A} / \mathrm{C}$ in the maintenance of the $3 \mathrm{D}$ genome organization, profoundly disturbed in $\mathrm{H}$ and RS cells, this interaction needs further investigation by $3 \mathrm{D}$ techniques.

Supplementary Information accompanies the paper on the Laboratory Investigation website (http://www.laboratoryinvestigation.org)

\section{ACKNOWLEDGMENTS}

We thank Mary Cheang, Biostatistician, for statistical analysis of data. The 3D Telomeres Technology platform (3DTT) and the TeloView are property to 3D Signatures and were used with the company's permission. We are grateful to receive research support from the Cole Foundation (NJ) and the Canadian Institutes of Health Research (SM; Grant MOP110982).

\section{DISCLOSURE/CONFLICT OF INTEREST}

SM is a founder of and currently a director, officer (CSO) and shareholder of 3D Signatures. HK is a member of the Scientific Advisory Board and shareholder of 3D Signatures. The remaining authors declare no conflict of interest.

1. MacLeod RA, Spitzer D, Bar-Am I, et al. Karyotypic dissection of Hodgkin's disease cell lines reveals ectopic subtelomeres and ribosomal DNA at sites of multiple jumping translocations and genomic amplification. Leukemia 2000;14:1803-1814.

2. Joos $\mathrm{S}$, Granzow M, Holtgreve-Grez $\mathrm{H}$, et al. Hodgkin's lymphoma cell lines are characterized by frequent aberrations on chromosomes $2 p$ and 9p including REL and JAK2. Int J Cancer 2003;103:489-495.

3. Mader A, Brüderlein S, Wegener S, et al. U-HO1, a new cell line derived from a primary refractory classical Hodgkin lymphoma. Cytogenet Genome Res 2007;119:204-210.

4. Hartmann S, Martin-Subero Jl, Gesk S, et al. Detection of genomic imbalances in microdissected Hodgkin and Reed-Sternberg cells of classical Hodgkin's lymphoma by array-based comparative genomic hybridization. Haematologica 2008;93:1318-1326.

5. Steidl C, Telenius A, Shah SP, et al. Genome-wide copy number analysis of Hodgkin Reed-Sternberg cells identifies recurrent imbalances with correlations to treatment outcome. Blood 2010;116:418-427.

6. Slovak ML, Bedell V, Hsu YH, et al. Molecular karyotypes of Hodgkin and Reed-Sternberg cells at disease onset reveal distinct copy number alterations in chemosensitive versus refractory Hodgkin lymphoma. Clin Cancer Res 2011;17:3443-3454.

7. Knecht $H$, Sawan B, Lichtensztejn D, et al. The 3D nuclear organization of telomeres marks the transition from Hodgkin to ReedSternberg cells. Leukemia 2009;23:565-573.

8. Guffei A, Sarkar R, Klewes $L$, et al. Dynamic chromosomal rearrangements in Hodgkin's lymphoma are due to ongoing threedimensional nuclear remodeling and breakage-bridge-fusion cycles. Haematologica 2010;95:2038-2046.

9. Knecht $H$, Righolt C, Mai S. Genomic instability: the driving force behind refractory/relapsing Hodgkin's lymphoma. Cancers 2013;5: 714-725.

10. Righolt C, Guffei A, Knecht $H$, et al. Differences in nuclear DNA organization between lymphocytes, Hodgkin and Reed-Sternberg cells revealed by structured illumination microscopy. J Cell Biochem 2014;115:1441-1448.

11. Righolt $C$, Knecht $H$, Mai S. DNA superresolution structure of Reed-Sternberg cells differs between long lasting remission versus relapsing Hodgkin's lymphoma patients. J Cell Biochem 2016;117: 1633-1637.

12. De Lange T. Shelterin: the protein complex that shapes and safeguards human telomeres. Genes Dev 2005;19:2100-2110.

13. Londoño-Vallejo JA, Wellinger RJ. Telomeres and telomerase dance to the rhythm of the cell cycle. Trends Biochem Sci 2012;37:391-399.

14. Lajoie V, Lemieux B, Sawan B, et al. LMP1 mediates multinuclearity through downregulation of shelterin proteins and formation of telomeric aggregates. Blood 2015;125:2101-2110.

15. Kis LL, Nishikawa J, Takahara M, et al. In vitro EBV-infected subline of $\mathrm{KMH} 2$, derived from Hodgkin lymphoma, expresses only EBNA-1, while CD40 ligand and IL-4 induce LMP-1 but not EBNA-2. Int J Cancer 2005;113:937-945.

16. Feuerhahn S, Chen LY, Luke B, et al. No DDRama at chromosome ends: TRF2 takes centre stage. Trends Biochem Sci 2015;40:275-285.

17. Wood AM, Rendtlew Danielsen JM, Lucas CA, et al. TRF2 and lamin A/C interact to facilitate the functional organization of chromosome ends. Nat Commun 2014;5:5467.

18. Lacoste $S$, Wiechec $E$, dos Santos Silva AG, et al. Chromosomal rearrangements after ex vivo Epstein-Barr virus (EBV) infection of human B cells. Oncogene 2010;29:503-515.

19. Kamranvar SA, Chen $X$, Masucci MG. Telomere dysfunction and activation of alternative lengthening of telomeres in B-lymphocytes infected by Epstein-Barr virus. Oncogene 2013;32:5522-5530.

20. Brousset $\mathrm{P}, \mathrm{Al}$ Saati $\mathrm{T}$, Chaouche $\mathrm{N}$, et al. Telomerase activity in reactive and neoplastic lymphoid tissue: infrequent detection of activity in Hodgkin's disease. Blood 1997;89:26-31.

21. Heine B, Hummel M, Demel G, et al. Hodgkin and Reed-Sternberg cells of classical Hodgkin's disease overexpress the telomerase RNA template (hTR). J Pathol 1999;188:139-145.

22. Ohshima K, Sugihara $M$, Haraoka $S$, et al. Possible immortalization of Hodgkin and Reed-Sternberg cells: telomerase expression, lengthening of telomere, and inhibition of apoptosis by NF-kappaB expression. Leuk Lymphoma 2001;42:639-647.

23. Louis SF, Vermolen BJ, Garini Y, et al. C-Myc induces chromosomal rearrangements through telomere and chromosome remodeling in the interphase nucleus. Proc Natl Acad Sci USA 2005;102:9613-9618.

24. Chuang TC, Moshir S, Garini $Y$, et al. The three-dimensional organization of telomeres in the nucleus of mammalian cells. BMC Biol 2004;2:12.

25. Schaefer LH, Schuster D, Herz H. Generalized approach for accelerated maximum likelihood based image restoration applied to threedimensional fluorescence microscopy. J Microsc 2001;204:99-107.

26. Vermolen BJ, Garini $Y$, Mai $S$, et al. Characterizing the threedimensional organization of telomeres. Cytometry A 2005;67: 144-150.

27. Mai S, Garini Y. The significance of telomeric aggregates in the interphase nuclei of tumor cells. J Cell Biochem 2006;97:904-915.

28. Knecht H, Sawan B, Lichtensztejn Z, et al. 3D telomere FISH defines LMP1 expressing Reed-Sternberg cells as end-stage cells with telomere-poor "ghost" nuclei. Lab Invest 2010;90:611-619.

29. Sarkar R, Guffei A, Vermolen BJ, et al. Alterations of centromere positions in nuclei of immortalized and malignant mouse lymphocytes. Cytometry A 2007;71:386-392.

30. Benarroch-Popivker D, Pisano S, Mendez-Bermudez A, et al. TRF2-mediated control of telomere DNA topology as a mechanism for chromosome-end protection. Mol Cell 2016;61:274-286.

31. Bronshtein I, Kepten E, Kanter I, et al. Loss of lamin A function increases chromatin dynamics in the nuclear interior. Nat Commun 2015;6:8044.

32. Nera B, Huang HS, Lai T, et al. Elevated levels of TRF2 induce telomeric ultrafine anaphase bridges and rapid telomere deletions. Nat Commun 2015;6:10132.

33. Dong W, Shen R, Wang $Q$, et al. Sp1 upregulates expression of TRF2 and TRF2 inhibition reduces tumorigenesis in human colorectal carcinoma cells. Cancer Biol Ther 2009:8:2166-2174.

34. Diehl MC, Idowu MO, Kimmelshue KN, et al. Elevated TRF2 in advanced breast cancers with short telomeres. Breast Cancer Res Treat 2011;127: 623-630. 
35. Bellon $M$, Datta $A$, Brown $M$, et al. Increased expression of telomere length regulating factors TRF1, TRF2 and TIN2 in patients with adult T-cell leukaemia. Int J Cancer 2006;119:2090-2097.

36. Biroccio A, Cherfils-Vicini J, Augereau A, et al. TRF2 inhibits a cellextrinsic pathway through which natural killer cells eliminate cancer cells. Nat Cell Biol 2013;15:818-828.

37. Celli GB, de Lange T. DNA processing is not required for ATM-mediated telomere damage response after TRF2 deletion. Nat Cell Biol 2005;7: 712-718.

38. Denchi EL, de Lange T. Protection of telomeres through independent control of ATM and ATR by TRF2 and POT1. Nature 2007;448: 1068-1071.

39. Lazzerini Denchi E, Celli G, et al. Hepatocytes with extensive telomere deprotection and fusion remain viable and regenerate liver mass through endoreduplication. Genes Dev 2006;20:2648-2653.

40. Davoli T, Denchi EL, de Lange T. Persistent telomere damage induces bypass of mitosis and tetraploidy. Cell 2010;141:81-93.

41. Alder JK, Barkauskas CE, Limjunyawong $\mathrm{N}$, et al. Telomere dysfunction causes alveolar stem cell failure. Proc Natl Acad Sci USA 2015;112: 5099-5104.

42. Luo $\mathrm{Z}$, Feng $\mathrm{X}$, Wang $\mathrm{H}$, et al. Mir-23a induces telomere dysfunction and cellular senescence by inhibiting TRF2 expression. Aging Cell 2015;14:391-399.
43. Khan G. Epstein-Barr virus, cytokines, and inflammation: a cocktail for the pathogenesis of Hodgkin's lymphoma? Exp Hematol 2006;34: 399-406.

44. Küppers R. The biology of Hodgkin's lymphoma. Nat Rev Cancer 2009;9:15-27.

45. Xu L, Blackburn EH. Human cancer cells harbour T-stumps, a distinct class of extremely short telomeres. Mol Cell 2007;28:315-327.

46. Chen X, Kamranvar SA, Masucci MG. Oxidative stress enables EpsteinBarr virus-induced B-cell transformation by posttranscriptional regulation of viral and cellular growth-promoting factors. Oncogene 2016;35: 3807-3816.

47. Xerri L, Birg F, Guigou V, et al. In situ expression of the IL-1-alpha and TNF-alpha genes by Reed-Sternberg cells in Hodgkin's disease. Int J Cancer 1992;50:689-693.

48. Caliò $A$, Zamò $A$, Ponzoni $M$, et al. Cellular senescence markers p16INK4a and p21CIP1/WAF are predictors of Hodgkin lymphoma outcome. Clin Cancer Res 2015;21:5164-5172.

49. Rodier F, Coppé JP, Patil CK, et al. Persistent DNA damage signalling triggers senescence-associated inflammatory cytokine secretion. Nat Cell Biol 2009;11:973-979.

50. Cesare AJ, Hayashi MT, Crabbe L, et al. The telomere deprotection response is functionally distinct from the genomic DNA damage response. Mol Cell 2013;51:141-155. 